\title{
A Stable and Conservative High Order Multi-block Method for the Compressible Navier-Stokes Equations
}

\author{
Jan Nordström $^{\mathrm{a}, \mathrm{b}, *}$, Jing Gong ${ }^{\mathrm{b}}$, Edwin van der Weide ${ }^{\mathrm{c}}$ \\ Magnus Svärd ${ }^{\mathrm{d}}$ \\ ${ }^{a}$ Department of Computational Physics, FOI, The Swedish Defence Research \\ Agency, SE-16490 Stockholm, Sweden \\ ${ }^{\mathrm{b}}$ Department of Information Technology, Scientific Computing, Uppsala \\ University, SE-751 05 Uppsala, Sweden \\ ${ }^{\mathrm{c}}$ Faculty of Engineering Technology, University of Twente, PO Box 217, 7500 AE \\ Enschede, The Netherlands \\ ${ }^{\mathrm{d}}$ Center of Mathematics for Applications, University of Oslo P.B 1053 Blindern \\ N-0316 Oslo, Norway
}

\begin{abstract}
A stable and conservative high order multi-block method for the time-dependent compressible Navier-Stokes equations has been developed. Stability and conservation are proved using summation-by-parts operators, weak interface conditions and the energy method. This development makes it possible to exploit the efficiency of the high order finite difference method for non-trivial geometries. The computational results corroborate the theoretical analysis.
\end{abstract}

Key words: Navier-Stokes, finite difference, high order, stability, conservation

\section{Introduction}

The high order finite difference method in combination with summation-byparts operators and weak boundary conditions can very efficiently and re-

* Corresponding author, Email adress: Jan.Nordstrom@foi.se

1 This work was done while the first two authors were visiting CTR, The Center for Turbulence Research at Stanford University. 
liably handle large problems on structured grids for reasonably smooth geometries. This has been shown in a sequence of papers, see for example $[8,17,11,12,14,19,21]$. The most recent papers $([19],[21])$ on this subject discuss the specific problem with farfield and no-slip boundaries.

In this paper we conclude the development by treating the similar but not identical problem with a stable and accurate coupling of blocks. The technology in the two papers [19],[21] together with the interface treatment in this paper will conclude the development of a high order accurate and truly stable multi-block finite difference method for the Navier-Stokes equations.

In the next phase of this development we will use the coupling technique developed in this paper and combine the high order finite difference method with the finite volume method in combination with unstructured grids which can more readily handle complex geometries. That development is ongoing, see for example [13],[3] and [15].

The development in this paper is the theoretical foundation for that work. The main challenge for multi-block methods is to manage the possible instability at the interfaces or block boundaries between sub-domains. We will focus on the coupling procedure at the interface and for the first time prove stability and conservation of a high order accurate multi-block finite difference method applied to the Navier-Stokes equations.

The rest of the paper is organized as follows. In the next section we present the symmetric constant coefficient form of the Navier-Stokes equations followed by a short discussion of well-posedness in section 3 . The formulation of the numerical method on a single domain is considered in section 4. The coupling procedure is the topic of section 5 and the numerical experiments are presented in section 6. Finally, conclusions are drawn in section 7.

\section{The Navier-Stokes equations}

The frozen coefficient time-dependent compressible Navier-Stokes equations in two-dimensions in non-conservative form are given by, see [1]

$$
\tilde{u}_{t}+A \tilde{u}_{x}+B \tilde{u}_{y}=C \tilde{u}_{x x}+D \tilde{u}_{x y}+E \tilde{u}_{x y}
$$

where $\tilde{u}=\left[\tilde{\rho}, \tilde{u}_{1}, \tilde{u}_{2}, \tilde{p}\right]^{T}$ and $A, B, C, D$, and $E$ are coefficient matrices. $\tilde{\rho}$ is the density, $\tilde{u}_{1}$ and $\tilde{u}_{2}$ are the velocities and $\tilde{p}$ is the pressure. To apply the energy method we must symmetrize (1). Following the technique of symmetrization developed in [1] and [16], a symmetric form of (1) is,

$$
u_{t}+\left(A_{1} u\right)_{x}+\left(A_{2} u\right)_{y}=\varepsilon\left[\left(B_{11} u_{x}+B_{12} u_{y}\right)_{x}+\left(B_{21} u_{x}+B_{22} u_{y}\right)_{y}\right],
$$


with $\varepsilon=1 / \operatorname{Re}, u=\left(c \tilde{\rho} /(\sqrt{\gamma} \rho), \tilde{u}_{1}, \tilde{u}_{2}, \rho \tilde{T} / \sqrt{\gamma(\gamma-1)}\right)^{T}$ and

$$
\begin{aligned}
& A_{1}=\left[\begin{array}{cccc}
u_{1} & \frac{c}{\sqrt{\gamma}} & 0 & 0 \\
\frac{c}{\sqrt{\gamma}} & u_{1} & 0 & \sqrt{\frac{\gamma-1}{\gamma}} c \\
0 & 0 & u_{1} & 0 \\
0 & \sqrt{\frac{\gamma-1}{\gamma}} c & 0 & u_{1}
\end{array}\right], A_{2}=\left[\begin{array}{cccc}
u_{2} & 0 & \frac{c}{\sqrt{\gamma}} & 0 \\
0 & u_{2} & 0 & 0 \\
\frac{c}{\sqrt{\gamma}} & 0 & u_{2} & \sqrt{\frac{\gamma-1}{\gamma}} c \\
0 & 0 & \sqrt{\frac{\gamma-1}{\gamma}} c & u_{2}
\end{array}\right], \\
& B_{11}=\left[\begin{array}{cccc}
0 & 0 & 0 & 0 \\
0 & \frac{\lambda+2 \mu}{\rho} & 0 & 0 \\
0 & 0 & \frac{\mu}{\rho} & 0 \\
0 & 0 & 0 & \frac{\gamma \mu}{\operatorname{Pr} \rho}
\end{array}\right], B_{12}=B_{21}=\left[\begin{array}{cccc}
0 & 0 & 0 & 0 \\
0 & 0 & \frac{\lambda+\mu}{2 \rho} & 0 \\
0 & \frac{\lambda+\mu}{2 \rho} & 0 & 0 \\
0 & 0 & 0 & 0
\end{array}\right], B_{22}=\left[\begin{array}{cccc}
0 & 0 & 0 & 0 \\
0 & \frac{\mu}{\rho} & 0 & 0 \\
0 & 0 & \frac{\lambda+2 \mu}{\rho} & 0 \\
0 & 0 & 0 & \frac{\gamma \mu}{\operatorname{Pr} \rho}
\end{array}\right] .
\end{aligned}
$$

In the vectors and matrices above we have used the temperature $\tilde{T}$, the ratio of the specific heats $\gamma=c_{p} / c_{v}$, the speed of sound $c$, the dynamic viscosity $\mu$, the bulk viscosity $\lambda$, the kinematic viscosity $\nu=\mu / \rho$, the Prandtl number $\operatorname{Pr}=\nu / \alpha$ ( $\alpha$ is the thermal diffusivity) and the Reynolds number $\operatorname{Re}=$ $\rho_{\infty} U_{\infty} L / \mu_{\infty}$. The infinity subscript denotes free stream conditions and $L$ is a characteristic length. Note again that the form of the matrices (Jacobians) above are obtained for the symmetrized frozen coefficient version of the NavierStokes equations.

Equation (2) can be rewritten in conservative form as

$$
u_{t}+F_{x}+G_{y}=0
$$

where

$$
\begin{aligned}
& F=A_{1} u-\varepsilon\left(B_{11} u_{x}+B_{12} u_{y}\right)=F^{I}-\varepsilon F^{V}, \\
& G=A_{2} u-\varepsilon\left(B_{21} u_{x}+B_{22} u_{y}\right)=G^{I}-\varepsilon G^{V} .
\end{aligned}
$$

$F^{I}$ and $G^{I}$ contain the inviscid terms and $F^{V}$ and $G^{V}$ the viscous terms.

\section{Well-posedness of the continuous problem}

To keep the algebraic complexity of the analysis as low as possible, we consider rectangular domains with cartesian coordinates. Applying the energy method 
to (3) on the domain $\Omega \in[-1,1] \times[0,1]$ we obtain

$$
\iint_{\Omega} u^{T} u_{t} d x d y+\iint_{\Omega} u^{T} F_{x} d x d y+\iint_{\Omega} u^{T} G_{y} d x d y=0 .
$$

By using the Green-Gauss theorem, equation (5) can be written as

$$
\begin{aligned}
\frac{d}{d t}\left(\|u\|^{2}\right)= & -\underbrace{\left.\int_{0}^{1} u^{T}\left(F^{I}-2 \varepsilon F^{V}\right)\right|_{x=1} d y}_{\text {East }}-\underbrace{\left.\int_{1}^{0} u^{T}\left(F^{I}-2 \varepsilon F^{V}\right)\right|_{x=-1} d y}_{\text {West }} \\
& -\underbrace{\left.\int_{-1}^{1} u^{T}\left(G^{I}-2 \varepsilon G^{V}\right)\right|_{y=0} d x}_{\text {South }}-\underbrace{\left.\int_{1}^{-1} u^{T}\left(G^{I}-2 \varepsilon G^{V}\right)\right|_{y=1} d x}_{\text {North }} \\
& -2 \varepsilon \iint_{\Omega}\left[\begin{array}{l}
u_{x} \\
u_{y}
\end{array}\right]^{\left[\begin{array}{ll}
B_{11} & B_{12} \\
B_{21} & B_{22}
\end{array}\right]}\left[\begin{array}{l}
u_{x} \\
u_{y}
\end{array}\right] d x d y .
\end{aligned}
$$

To have a bounded energy growth, the boundary terms (East, West, North and South) must be bounded using the correct number and form of boundary conditions. That is the topic in papers [19],[21] and it is not discussed further here. The contribution from the integral term is negative semi-definite since the matrix $B=\left[\begin{array}{llll}B_{11} & B_{12} ; B_{21} & B_{22}\end{array}\right]$ is positive semi-definite.

We summarize the result for the continuous problem (2)-(4) in the following proposition.

Proposition 3.1 The continuous problem (2)-(4) is well posed if $i)$ the correct number and form of the boundary conditions are used to bound the boundary terms and ii) the matrix $B=\left[\begin{array}{lll}B_{11} & B_{12} ; B_{21} & B_{22}\end{array}\right]$ is positive semi-definite.

Remark The $n$-dimensional Navier-Stokes equations require $\mathrm{n}$ boundary conditions at an inflow boundary and $n-1$ at an outflow boundary. In this case (two-dimensions) we need four boundary conditions an inflow boundary and three at an outflow boundary, see for example [18],[6],[16].

\section{Stability on a single domain}

Consider the computational domain with a Cartesian mesh of $(M+1) \times(N+1)$ points. Let the $k$-th element of the continuous variable $u$ at the structured grid point $\left(x_{i}, y_{j}\right)$ be $u(i, j, k)(0 \leq k \leq 3)$. The finite difference approximation of $u(i, j, k)$ is collected in a global vector $\mathbf{v}$ such that $v[4 i(N+1)+4 j+k]=$ $u(i, j, k)(0 \leq i \leq M, 0 \leq j \leq N$ and $0 \leq k \leq 3)$. Let $\mathbf{v}_{x}$ and $\mathbf{v}_{y}$ be 
approximations of $u_{x}$ and $u_{y}$. By using the finite difference method developed in $[8,17,11,12,14,19,21]$ a semi-discrete approximation of equation (3) can be written as

$$
\mathbf{v}_{t}+D_{x} \mathbf{F}+D_{y} \mathbf{G}=0
$$

where $D_{x}=P_{x}^{-1} Q_{x} \otimes I_{y} \otimes I_{4}$ and $D_{y}=I_{x} \otimes P_{y}^{-1} Q_{y} \otimes I_{4}$ are first derivative operators in $x$-, and $y$ - directions, respectively. $I_{x}$ and $I_{y}$ are the identity matrices of size $(M+1) \times(M+1)$ and $(N+1) \times(N+1)$. Moreover,

$$
\mathbf{F}=\mathbf{F}^{I}-\varepsilon \mathbf{F}^{V}, \quad \mathbf{G}=\mathbf{G}^{I}-\varepsilon \mathbf{G}^{V},
$$

with

$$
\begin{aligned}
\mathbf{F}^{I}=\left(I_{x} \otimes I_{y} \otimes A_{1}\right) \mathbf{v}, & \mathbf{F}^{V}=\left(I_{x} \otimes I_{y} \otimes B_{11}\right) \mathbf{v}_{x}+\left(I_{x} \otimes I_{y} \otimes B_{12}\right) \mathbf{v}_{y}, \\
\mathbf{G}^{I}=\left(I_{x} \otimes I_{y} \otimes A_{2}\right) \mathbf{v}, & \mathbf{G}^{V}=\left(I_{x} \otimes I_{y} \otimes B_{21}\right) \mathbf{v}_{x}+\left(I_{x} \otimes I_{y} \otimes B_{22}\right) \mathbf{v}_{y},
\end{aligned}
$$

and $\mathbf{v}_{x}=D_{x} \mathbf{v}, \mathbf{v}_{y}=D_{y} \mathbf{v}$. Let $\bar{P}=P_{x} \otimes P_{y}$ and multiply equation (7) with $\mathbf{v}^{T}\left(\bar{P} \otimes I_{4}\right)$. (This is the discrete equivalent of multiplying (3) with $v^{T}$ and integrating over the computational domain to get the energy estimate (6)). This leads to

$$
\mathbf{v}^{T}\left(\bar{P} \otimes I_{4}\right) \mathbf{v}_{t}+\mathbf{v}^{T}\left(Q_{x} \otimes P_{y} \otimes I_{4}\right) \mathbf{F}+\mathbf{v}^{T}\left(P_{x} \otimes Q_{y} \otimes I_{4}\right) \mathbf{G}=0
$$

By adding the transpose of equation (8) to itself and using the SBP relations

$$
Q_{x}+Q_{x}^{T}=\operatorname{diag}(-1,0, \ldots, 0,1), \quad Q_{y}+Q_{y}^{T}=\operatorname{diag}(-1,0, \ldots, 0,1)
$$

we can write the result as

$$
\frac{d}{d t}\left(\|\mathbf{v}\|_{\bar{P} \otimes I_{4}}^{2}\right)=-\mathrm{IT}+\varepsilon \mathrm{VT} .
$$

The inviscid term IT in (10) is

$$
\begin{aligned}
\mathrm{IT}= & \mathbf{v}^{T}\left(Q_{x} \otimes P_{y} \otimes I_{4}\right) \mathbf{F}^{I}+\left(\mathbf{F}^{I}\right)^{T}\left(Q_{x}^{T} \otimes P_{y} \otimes I_{4}\right) \mathbf{v}+ \\
& \mathbf{v}^{T}\left(P_{x} \otimes Q_{y} \otimes I_{4}\right) \mathbf{G}^{I}+\left(\mathbf{G}^{I}\right)^{T}\left(P_{x} \otimes Q_{y}^{T} \otimes I_{4}\right) \mathbf{v} \\
= & \underbrace{\mathbf{v}_{E}^{T}\left(P_{y} \otimes I_{4}\right) \mathbf{F}_{E}^{I}}_{\text {East }}-\underbrace{\mathbf{v}_{W}^{T}\left(P_{y} \otimes I_{4}\right) \mathbf{F}_{W}^{I}}_{\text {West }}-\underbrace{\mathbf{v}_{S}^{T}\left(P_{x} \otimes I_{4}\right) \mathbf{G}_{S}^{I}}_{\text {South }}+\underbrace{\mathbf{v}_{N}^{T}\left(P_{x} \otimes I_{4}\right) \mathbf{G}_{N}^{I}}_{\text {North }}
\end{aligned}
$$


The viscous term VT in (10) can be written as

$$
\begin{aligned}
\mathrm{VT}= & \mathbf{v}^{T}\left(Q_{x} \otimes P_{y} \otimes I_{4}\right) \mathbf{F}^{V}+\left(\mathbf{F}^{V}\right)^{T}\left(Q_{x}^{T} \otimes P_{y} \otimes I_{4}\right) \mathbf{v}+ \\
& \mathbf{v}^{T}\left(P_{x} \otimes Q_{y} \otimes I_{4}\right) \mathbf{G}^{V}+\left(\mathbf{G}^{V}\right)^{T}\left(P_{x} \otimes Q_{y} \otimes I_{4}\right)^{T} \mathbf{v} \\
= & 2 \underbrace{\mathbf{v}_{E}^{T}\left(P_{y} \otimes I_{4}\right) \mathbf{F}_{E}^{V}}_{\text {East }}-2 \underbrace{\mathbf{v}_{W}^{T}\left(P_{y} \otimes I_{4}\right) \mathbf{F}_{W}^{V}}_{\text {West }}-2 \underbrace{\mathbf{v}_{S}^{T}\left(P_{x} \otimes I_{4}\right) \mathbf{G}_{S}^{V}}_{\text {South }} \\
& +2 \underbrace{\mathbf{v}_{N}^{T}\left(P_{x} \otimes I_{4}\right) \mathbf{G}_{N}^{V}}_{\text {North }}-2\left[\begin{array}{c}
\mathbf{v}_{x} \\
\mathbf{v}_{y}
\end{array}\right]^{T}\left[\begin{array}{l}
\bar{P} \otimes B_{11} \bar{P} \otimes B_{12} \\
\bar{P} \otimes B_{21} \bar{P} \otimes B_{22}
\end{array}\right]\left[\begin{array}{l}
\mathbf{v}_{x} \\
\mathbf{v}_{y}
\end{array}\right] .
\end{aligned}
$$

An expanded version of equation (10) using the relations above becomes

$$
\begin{aligned}
& \frac{d}{d t}\left(\|\mathbf{v}\|_{\bar{P} \otimes I_{4}}^{2}\right)=-\underbrace{\mathbf{v}_{E}^{T}\left(P_{y} \otimes I_{4}\right)\left(\mathbf{F}_{E}^{I}-2 \varepsilon \mathbf{F}_{E}^{V}\right)}_{\text {East }}+\underbrace{\mathbf{v}_{W}^{T}\left(P_{y} \otimes I_{4}\right)\left(\mathbf{F}_{W}^{I}-2 \varepsilon \mathbf{F}_{W}^{V}\right)}_{\text {West }} \\
& +\underbrace{\mathbf{v}_{S}^{T}\left(P_{x} \otimes I_{4}\right)\left(\mathbf{G}_{S}^{I}-2 \varepsilon \mathbf{G}_{S}^{V}\right)}_{\text {South }}-\underbrace{\mathbf{v}_{N}^{T}\left(P_{x} \otimes I_{4}\right)\left(\mathbf{G}_{N}^{I}-2 \varepsilon \mathbf{G}_{N}^{V}\right)}_{\text {North }} \\
& -2 \varepsilon\left[\begin{array}{l}
\mathbf{v}_{x} \\
\mathbf{v}_{y}
\end{array}\right]^{T}\left[\begin{array}{ll}
\bar{P} \otimes B_{11} & \bar{P} \otimes B_{12} \\
\bar{P} \otimes B_{21} & \bar{P} \otimes B_{22}
\end{array}\right]\left[\begin{array}{l}
\mathbf{v}_{x} \\
\mathbf{v}_{y}
\end{array}\right] \text {. }
\end{aligned}
$$

Note that for square matrices $\bar{P}$ and $B_{11}$ (or $B_{12}, B_{21}$ and $B_{22}$ ) the Kronecker product $\bar{P} \otimes B_{11}$ and $B_{11} \otimes \bar{P}$ are even permutation similar, that is, there exists a permutation matrix $\Phi$ such that $\bar{P} \otimes B_{11}=\Phi^{T}\left(B_{11} \otimes \bar{P}\right) \Phi$, see [7] for details. Equation (13) can therefore be written

$$
\frac{d}{d t}\left(\|\mathbf{v}\|_{\bar{P} \otimes I_{4}}^{2}\right)=\mathrm{BT}-2 \varepsilon\left[\begin{array}{l}
\mathbf{w}_{x} \\
\mathbf{w}_{y}
\end{array}\right]^{T}\left[\begin{array}{ll}
B_{11} & B_{12} \\
B_{21} & B_{22}
\end{array}\right] \otimes \bar{P}\left[\begin{array}{l}
\mathbf{w}_{x} \\
\mathbf{w}_{y}
\end{array}\right]
$$

where BT collect all the boundary terms in (13) and $\mathbf{w}_{x}=\Phi \mathbf{v}_{x}, \mathbf{w}_{y}=\Phi \mathbf{v}_{y}$.

Exactly similar to the continuous case, a bounded energy growth in (14) require boundedness in terms of given data of the boundary terms (East, West, North and South). Again, that is dealt with in the papers [19],[21]. The contribution from the integral term in (14) is negative semi-definite since the matrices $\bar{P}$ and $B=\left[\begin{array}{llll}B_{11} & B_{12} ; B_{21} & B_{22}\end{array}\right]$ are positive semi-definite.

Exactly similar to the continuous case, we summarize the result for the semidiscrete single domain problem (7) in the following proposition.

Proposition 4.1 The semi-discrete problem (7) is stable if i) the boundary terms $B T$ are limited by given data and ii) the matrices $\bar{P}$ and the matrix $B=\left[\begin{array}{llll}B_{11} & B_{12} ; & B_{21} & B_{22}\end{array}\right]$ are positive semi-definite. 


\section{$5 \quad$ Stable and conservative interface conditions}

We consider a computational domain consisting of two sub-domains and a common interface at $x=0$, see Figure 1 . Let $\mathbf{u}$ and $\mathbf{v}$ be the unknowns in the left and right sub-domain, respectively, and introduce the superscripts ${ }^{L}$ and $R$ to identify the left and right sub-domains.

The semi-discrete approximation of (2) on the two sub-domains with an interface can be written

$$
\begin{aligned}
\mathbf{u}_{t}+D_{x}^{L} \mathbf{F}^{L}+D_{y}^{L} \mathbf{G}^{L} & =\left(M^{L}\right)^{-1}\left(\bar{\Sigma}_{1}^{L}\left[\mathbf{u}_{I}-\mathbf{v}_{I}\right]+\bar{\Sigma}_{2}^{L}\left[\left(\mathbf{F}^{V}\right)_{I}^{L}-\left(\mathbf{F}^{V}\right)_{I}^{R}\right]\right) \\
\mathbf{v}_{t}+D_{x}^{R} \mathbf{F}^{R}+D_{y}^{R} \mathbf{G}^{R} & =\left(M^{R}\right)^{-1}\left(\bar{\Sigma}_{1}^{R}\left[\mathbf{v}_{I}-\mathbf{u}_{I}\right]+\bar{\Sigma}_{2}^{R}\left[\left(\mathbf{F}^{V}\right)_{I}^{R}-\left(\mathbf{F}^{V}\right)_{I}^{L}\right]\right)
\end{aligned}
$$

where the matricies $E^{L}, E^{R}$ picks out the parts of the vectors residing at the interface such that for example $\mathbf{u}_{I}=E^{L} \mathbf{u}, \mathbf{v}_{I}=E^{R} \mathbf{v}$. In the following, the subscript $I$ indicates that the quantity resides on the interface. We also have the definitions:

$$
\begin{aligned}
D_{x}^{L} & =\left(P_{x}^{L}\right)^{-1} Q_{x}^{L} \otimes I_{y}^{L} \otimes I_{4}, & & D_{y}^{L}=I_{x}^{L} \otimes\left(P_{y}^{L}\right)^{-1} Q_{y}^{L} \otimes I_{4}, \\
D_{x}^{R} & =\left(P_{x}^{R}\right)^{-1} Q_{x}^{R} \otimes I_{y}^{R} \otimes I_{4}, & & D_{y}^{R}=I_{x}^{R} \otimes\left(P_{y}^{R}\right)^{-1} Q_{y}^{R} \otimes I_{4}, \\
M^{L} & =P_{x}^{L} \otimes P_{y}^{L} \otimes I_{4}, & M^{R} & =P_{x}^{R} \otimes P_{y}^{R} \otimes I_{4}, \\
\bar{\Sigma}_{1}^{L} & =\left(E^{L}\right)^{T} P_{y}^{L} \otimes \Sigma_{1}^{L}, & \bar{\Sigma}_{2}^{L} & =\left(E^{L}\right)^{T} P_{y}^{L} \otimes \Sigma_{2}^{L}, \\
\bar{\Sigma}_{1}^{R} & =\left(E^{R}\right)^{T} P_{y}^{R} \otimes \Sigma_{1}^{R}, & \bar{\Sigma}_{2}^{R} & =\left(E^{R}\right)^{T} P_{y}^{R} \otimes \Sigma_{2}^{R} .
\end{aligned}
$$

Note that the outer boundary conditions are neglected in this analysis, or phrased differently, they are considered separately, see [19],[21].

Applying the energy method to (15a) and (15b) yields

$$
\frac{d}{d t}\left(\|\mathbf{u}\|_{M^{L}}^{2}+\|\mathbf{v}\|_{M^{R}}^{2}\right)+2 \varepsilon \operatorname{Diss}=\mathbf{w}_{I}^{T} M \mathbf{w}_{I}
$$

where $\mathbf{w}_{I}=\left[\mathbf{u}_{I}, \mathbf{v}_{I},\left(\mathbf{u}_{x}\right)_{I},\left(\mathbf{v}_{x}\right)_{I},\left(\mathbf{u}_{y}\right)_{I},\left(\mathbf{v}_{y}\right)_{I}\right]^{T}$ and

$$
\begin{aligned}
& \operatorname{Diss}=\left[\begin{array}{c}
\mathbf{u}_{x} \\
\mathbf{u}_{y}
\end{array}\right]^{T}\left[\begin{array}{cc}
P_{x}^{L} \otimes P_{y}^{L} \otimes B_{11} & P_{x}^{L} \otimes P_{y}^{L} \otimes B_{12} \\
P_{x}^{L} \otimes P_{y}^{L} \otimes B_{21} & P_{x}^{L} \otimes P_{y}^{L} \otimes B_{22}
\end{array}\right]\left[\begin{array}{l}
\mathbf{u}_{x} \\
\mathbf{u}_{y}
\end{array}\right]+ \\
& {\left[\begin{array}{l}
\mathbf{v}_{x} \\
\mathbf{v}_{y}
\end{array}\right]^{T}\left[\begin{array}{cc}
P_{x}^{R} \otimes P_{y}^{R} \otimes B_{11} & P_{x}^{R} \otimes P_{y}^{R} \otimes B_{12} \\
P_{x}^{R} \otimes P_{y}^{R} \otimes B_{21} & P_{x}^{R} \otimes P_{y}^{R} \otimes B_{22}
\end{array}\right]\left[\begin{array}{l}
\mathbf{v}_{x} \\
\mathbf{v}_{y}
\end{array}\right] .}
\end{aligned}
$$

The matrix $M$ in (16) determines the stability of the interface treatment. $M$ is 
symmetric and the elements of $M$ are

$$
\begin{array}{ll}
M_{11}=P_{y}^{L} \otimes\left(-A_{1}+\Sigma_{1}^{L}+\left(\Sigma_{1}^{L}\right)^{T}\right), & M_{12}=P_{y}^{L} \otimes-\Sigma_{1}^{L}+P_{y}^{R} \otimes-\left(\Sigma_{1}^{R}\right)^{T}, \\
M_{13}=P_{y}^{L} \otimes\left(\varepsilon I_{4}+\Sigma_{2}^{L}\right) B_{11}, & M_{14}=P_{y}^{L} \otimes-\Sigma_{2}^{L} B_{11}, \\
M_{15}=P_{y}^{L} \otimes\left(\varepsilon I_{4}+\Sigma_{2}^{L}\right) B_{12}, & M_{16}=P_{y}^{L} \otimes-\Sigma_{2}^{L} B_{12}, \\
M_{22}=P_{y}^{R} \otimes\left(A_{1}+\Sigma_{1}^{R}+\left(\Sigma_{1}^{R}\right)^{T}\right), & M_{23}=P_{y}^{R} \otimes-\Sigma_{2}^{R} B_{11}, \\
M_{24}=P_{y}^{L} \otimes\left(-\varepsilon I_{4}+\Sigma_{2}^{R}\right) B_{11}, & M_{25}=P_{y}^{L} \otimes-\Sigma_{2}^{R} B_{12}, \\
M_{26}=P_{y}^{R} \otimes\left(-\varepsilon I_{4}+\Sigma_{2}^{R}\right) B_{12}, & M_{33}=M_{34}=M_{35}=M_{36}=0, \\
M_{44}=M_{45}=M_{46}=0, & M_{55}=M_{56}=M_{66}=0 .
\end{array}
$$

Notice that the matrix $M$ in its present form is indefinite.

In order to construct a symmetric semi-definite negative matrix on the right hand side of equation (16) we must "borrow" interface terms from Diss on the left hand side, see [2]. The term Diss can be written as

$$
\begin{array}{r}
\text { Diss }=\widetilde{D i s s}+\alpha^{L} p^{L}\left[\begin{array}{l}
\left(\mathbf{u}_{x}\right)_{I} \\
\left(\mathbf{u}_{y}\right)_{I}
\end{array}\right]^{T}\left[\begin{array}{ll}
P_{y}^{L} \otimes B_{11} & P_{y}^{L} \otimes B_{12} \\
P_{y}^{L} \otimes B_{21} & P_{y}^{L} \otimes B_{22}
\end{array}\right]\left[\begin{array}{l}
\left(\mathbf{u}_{x}\right)_{I} \\
\left(\mathbf{u}_{y}\right)_{I}
\end{array}\right] \\
+\beta^{R} p^{R}\left[\begin{array}{l}
\left(\mathbf{v}_{x}\right)_{I} \\
\left(\mathbf{v}_{y}\right)_{I}
\end{array}\right]^{T}\left[\begin{array}{ll}
P_{y}^{R} \otimes B_{11} & P_{y}^{R} \otimes B_{12} \\
P_{y}^{R} \otimes B_{21} & P_{y}^{R} \otimes B_{22}
\end{array}\right]\left[\begin{array}{l}
\left(\mathbf{v}_{x}\right)_{I} \\
\left(\mathbf{v}_{y}\right)_{I}
\end{array}\right]
\end{array}
$$

where $p^{L}=\left(P_{x}^{L}\right)_{M, M}, p^{R}=\left(P_{x}^{R}\right)_{1,1}$ and

$$
\begin{aligned}
\widetilde{D i s s}= & {\left[\begin{array}{l}
\mathbf{u}_{x} \\
\mathbf{u}_{y}
\end{array}\right]^{T}\left[\begin{array}{ll}
\widetilde{P_{x}^{L}} \otimes P_{y}^{L} \otimes B_{11} & \widetilde{P_{x}^{L}} \otimes P_{y}^{L} \otimes B_{12} \\
\widetilde{P_{x}^{L}} \otimes P_{y}^{L} \otimes B_{21} & \widetilde{P_{x}^{L}} \otimes P_{y}^{L} \otimes B_{22}
\end{array}\right]\left[\begin{array}{l}
\mathbf{u}_{x} \\
\mathbf{u}_{y}
\end{array}\right]+} \\
& {\left[\begin{array}{c}
\mathbf{v}_{x} \\
\mathbf{v}_{y}
\end{array}\right]^{T}\left[\begin{array}{ll}
\widetilde{P_{x}^{R}} \otimes P_{y}^{R} \otimes B_{11} & \widetilde{P_{x}^{R}} \otimes P_{y}^{R} \otimes B_{12} \\
\widetilde{P_{x}^{R}} \otimes P_{y}^{R} \otimes B_{21} & \widetilde{P_{x}^{R}} \otimes P_{y}^{R} \otimes B_{22}
\end{array}\right]\left[\begin{array}{l}
\mathbf{v}_{x} \\
\mathbf{v}_{y}
\end{array}\right] . }
\end{aligned}
$$

The modified norms in $\widetilde{D i s s}$ are $\widetilde{P_{x}^{L}}=P_{x}^{L}-\operatorname{diag}\left(0, . ., \alpha^{L} p^{L}\right)$ and $\widetilde{P_{x}^{R}}=P_{x}^{R}-$ $\operatorname{diag}\left(\beta^{R} p^{R}, 0 . ., 0\right)$. Note that with $0<\alpha^{L}, \beta^{R} \leq 1$, then $\widetilde{P_{x}^{L}} \geq 0$ and $\widetilde{P_{x}^{R}} \geq 0$ and hence $\widetilde{D i s s} \geq 0$.

As a result, the modified version of equation (16) can be written as

$$
\frac{d}{d t}\left(\|\mathbf{u}\|_{M^{L}}^{2}+\|\mathbf{v}\|_{M^{R}}^{2}\right)+2 \varepsilon \widetilde{\operatorname{Diss}}=\mathbf{w}_{I}^{T} \widetilde{M} \mathbf{w}_{I},
$$

where $\widetilde{M}$ plays the role of $M$ except that the zero elements in $M$ are replaced by

$$
\begin{array}{ll}
M_{33}=-2 \varepsilon \alpha^{L} p^{L} P_{y}^{L} \otimes B_{11}, & M_{35}=-2 \varepsilon \alpha^{L} p^{L} P_{y}^{L} \otimes B_{12}, \\
M_{44}=-2 \varepsilon \beta^{R} p^{R} P_{y}^{R} \otimes B_{11}, & M_{46}=-2 \varepsilon \beta^{R} p^{R} P_{y}^{R} \otimes B_{12}, \\
M_{55}=-2 \varepsilon \alpha^{L} p^{L} P_{y}^{L} \otimes B_{22}, & M_{66}=-2 \varepsilon \beta^{R} p^{R} P_{y}^{R} \otimes 2 B_{22}, \\
M_{53}=M_{35}^{T}, & M_{64}=M_{46}^{T} .
\end{array}
$$




\subsection{Conservation conditions}

Before considering the stability, we investigate the conservation properties at the interface. Let $\varphi$ be a smooth test function $\left(\varphi_{I}^{L}=\varphi_{I}^{R}=\varphi_{I}\right)$. Multiplying equations (15a) and (15b) by $\left(\varphi^{L}\right)^{T} M^{L}$ and $\left(\varphi^{R}\right)^{T} M^{R}$ respectively and using the SBP relations (9) leads to

$$
\begin{aligned}
& \left(\varphi^{L}\right)^{T} M^{L} \mathbf{u}_{t}+\left(\varphi^{R}\right)^{T} M^{R} \mathbf{v}_{t}-\left(\varphi_{x}^{T} M \mathbf{F}+\varphi_{y}^{T} M \mathbf{G}\right)^{L}-\left(\varphi_{x}^{T} M \mathbf{F}+\varphi_{y}^{T} M \mathbf{G}\right)^{R}= \\
& \varphi_{I}^{T}\left[-\left(P_{y}^{L} \otimes A_{1}\right) \mathbf{u}_{I}+\left(P_{y}^{R} \otimes A_{1}\right) \mathbf{v}_{I}+\left(P_{y}^{L} \otimes \Sigma_{1}^{L}-P_{y}^{R} \otimes \Sigma_{1}^{R}\right)\left(\mathbf{u}_{I}-\mathbf{v}_{I}\right)\right]+ \\
& \varphi_{I}^{T}\left[\left(P_{y}^{L} \otimes \varepsilon I_{4}\right)\left(\mathbf{F}_{I}^{V}\right)^{L}-\left(P_{y}^{R} \otimes \varepsilon I_{4}\right)\left(\mathbf{F}_{I}^{V}\right)^{R}+\left(P_{y}^{L} \otimes \Sigma_{1}^{L}-P_{y}^{R} \otimes \Sigma_{1}^{R}\right)\left(\left(\mathbf{F}_{I}^{V}\right)^{L}-\left(\mathbf{F}_{I}^{V}\right)^{R}\right)\right]
\end{aligned}
$$

As usual we have neglected the boundary terms. If $P_{y}^{L}=P_{y}^{R}$, the conditions

$$
\Sigma_{1}^{R}=\Sigma_{1}^{L}-A_{1}, \quad \Sigma_{2}^{R}=\Sigma_{2}^{L}+\varepsilon I_{4},
$$

cancel the interface terms (the right-hand side) in (18) and lead to a conservative scheme.

Remark The conservation conditions (19) are a subset of the resulting stability conditions, see $[12,14]$ for more details.

\subsection{Stability conditions}

Inserting $P_{y}^{L}=P_{y}^{R}=P_{y}$ and the conservation conditions (19) into (17) results in

$$
\left.\begin{array}{l}
\frac{d}{d t}\left(\|\mathbf{u}\|_{M^{L}}^{2}+\|\mathbf{v}\|_{M^{R}}^{2}\right)+2 \varepsilon \widetilde{\mathrm{Diss}} \\
\Psi \mathbf{v} \\
\Phi \mathbf{u}_{x} \\
\Psi \mathbf{v}_{x} \\
\Phi \mathbf{u}_{y} \\
\Psi \mathbf{v}_{y}
\end{array}\right]_{I}^{T}\left[\begin{array}{cccccc}
N_{11} & -N_{11} & N_{13} & N_{14} & N_{15} & N_{16} \\
-N_{11} & N_{11} & -N_{13} & -N_{14} & -N_{15} & -N_{16} \\
N_{13}^{T} & -N_{13}^{T} & N_{33} & 0 & N_{35} & 0 \\
N_{14}^{T} & -N_{14}^{T} & 0 & N_{44} & 0 & N_{46} \\
N_{15}^{T} & -N_{15}^{T} & N_{35}^{T} & 0 & N_{55} & 0 \\
N_{16}^{T} & -N_{16}^{T} & 0 & N_{46}^{T} & 0 & N_{66}
\end{array}\right] \otimes P_{y}\left[\begin{array}{c}
\Phi \mathbf{u} \\
\Psi \mathbf{v} \\
\Phi \mathbf{u}_{x} \\
\Psi \mathbf{v}_{x} \\
\Phi \mathbf{u}_{y} \\
\Psi \mathbf{v}_{y}
\end{array}\right]_{I}
$$

The permutation matrices $\Phi$ and $\Psi$ are defined in Section 4 and

$$
\begin{array}{llll}
\left.N_{11}=A_{1}-\Sigma_{1}^{L}-\left(\Sigma_{1}^{L}\right)^{T}\right), & N_{13}=-\left(\varepsilon I_{4}+\Sigma_{2}^{L}\right) B_{11}, & & N_{14}=\Sigma_{2}^{L} B_{11}, \\
N_{15}=-\left(\varepsilon I_{4}+\Sigma_{2}^{L}\right) B_{12}, & N_{16}=\Sigma_{2}^{L} B_{12} . & N_{33}=2 \varepsilon \alpha^{L} p^{L} B_{11}, \\
N_{35}=2 \varepsilon \alpha^{L} p^{L} B_{12}, & N_{44}=2 \varepsilon \beta^{R} p^{R} B_{11}, & N_{46}=2 \varepsilon \beta^{R} p^{R} B_{12}, \\
N_{55}=2 \varepsilon \alpha^{L} p^{L} B_{22}, & N_{66}=2 \varepsilon \beta^{R} p^{R} B_{22} . &
\end{array}
$$


Remark The condition $P_{y}^{L}=P_{y}^{R}$ implies that the same SBP operators should be used in the $y$-direction in both sub-domains. This restriction can be removed, and that will be the topic in a future paper.

A bounded energy require a positive semi-definite matrix $N$. To simplify the algebra we introduce a transformation matrix $S$ such that $S^{T} S=I$ and

$$
S=\left[\begin{array}{cccccc}
\frac{1}{\sqrt{2}} I_{4} & \frac{1}{\sqrt{2}} I_{4} & 0 & 0 & 0 & 0 \\
0 & 0 & I_{4} & 0 & 0 & 0 \\
0 & 0 & 0 & 0 & I_{4} & 0 \\
0 & 0 & 0 & I_{4} & 0 & 0 \\
0 & 0 & 0 & 0 & 0 & I_{4} \\
\frac{1}{\sqrt{2}} I_{4} & -\frac{1}{\sqrt{2}} I_{4} & 0 & 0 & 0 & 0
\end{array}\right], \hat{N}=S N S^{T}=\left[\begin{array}{cccccc}
0 & 0 & 0 & 0 & 0 & 0 \\
0 & N_{33} & N_{35} & 0 & 0 & \sqrt{2} N_{13} \\
0 & N_{35}^{T} & N_{55} & 0 & 0 & \sqrt{2} N_{15} \\
0 & 0 & 0 & N_{44} & N_{46} & \sqrt{2} N_{14} \\
0 & 0 & 0 & N_{46}^{T} & N_{66} & \sqrt{2} N_{16} \\
0 & \sqrt{2} N_{13}^{T} & \sqrt{2} N_{15}^{T} & \sqrt{2} N_{14}^{T} & \sqrt{2} N_{16}^{T} & 2 N_{11}
\end{array}\right]
$$

To simplify the matrix $\hat{N}$ we introduce

$$
\alpha=\alpha^{L} p^{L}, \quad \beta=\beta^{R} p^{R}, \quad \Sigma_{2}^{L}=-\varepsilon \Delta, \quad \Sigma_{1}^{L}=\Sigma_{1 I}^{L}+\varepsilon \Sigma_{1 V}^{L},
$$

where we choose $\Delta$ to be diagonal. This means that $\hat{N}$ can be split into an inviscid part $\hat{N}_{I}$ and a viscous part $\hat{N}_{V}$, we get

$$
\hat{N}=\underbrace{\left[\begin{array}{cc}
\mathbf{0}_{20,20} & \mathbf{0}_{20,4} \\
\mathbf{0}_{4,20} & 2\left(A_{1}-\Sigma_{1 I}^{L}-\left(\Sigma_{1 I}^{L}\right)^{T}\right)
\end{array}\right]}_{\hat{N}_{I}}+\underbrace{\left[\begin{array}{cccc}
\mathbf{0}_{4,4} & \mathbf{0}_{4,8} & \mathbf{0}_{4,8} & \mathbf{0}_{4,4} \\
\mathbf{0}_{8,4} & 2 \alpha K_{11} & \mathbf{0}_{8,8} & \sqrt{2} K_{13} \\
\mathbf{0}_{8,4} & \mathbf{0}_{8,8} & 2 \beta K_{11} & \sqrt{2} K_{23} \\
\mathbf{0}_{4,4} \sqrt{2} K_{13}^{T} \sqrt{2} K_{23}^{T} & 2 K_{33}
\end{array}\right]}_{\hat{N}_{V}}
$$

where $K_{33}=-\left(\Sigma_{1 V}+\left(\Sigma_{1 V}\right)^{T}\right.$ and

$$
K_{11}=\left[\begin{array}{ll}
B_{11} & B_{12} \\
B_{21} & B_{22}
\end{array}\right], K_{13}=\left[\begin{array}{l}
\left(\Delta-I_{4}\right) B_{11} \\
\left(\Delta-I_{4}\right) B_{12}
\end{array}\right], K_{23}=\left[\begin{array}{c}
-\Delta B_{11} \\
-\Delta B_{12}
\end{array}\right] .
$$

The subscripts on $\mathbf{0}$ in (22) indicate the size of the block.

The condition for $\hat{N}_{I}$ in equation (22) to be positive semi-definite is

$$
A_{1}-\Sigma_{1 I}^{L}-\left(\Sigma_{1 I}^{L}\right)^{T} \geq 0
$$

If $A_{1}$ is rewritten as $A_{1}=X^{T} \Lambda X=X^{T} \Lambda^{+} X+X^{T} \Lambda^{-} X=A_{1}^{+}+A_{1}^{-}$where $\Lambda^{+}=\operatorname{diag}\left(\max \left(\lambda_{i}, 0\right)\right), \Lambda^{-}=\operatorname{diag}\left(\min \left(\lambda_{i}, 0\right)\right)$ and $\lambda_{i}$ are the eigenvalues of $A_{1}$, we find that (23) is satisfied if

$$
\Sigma_{1 I}^{L}+\left(\Sigma_{1 I}^{L}\right)^{T} \leq A_{1}^{-}
$$


Next we turn to the more difficult analysis of the definiteness of $\hat{N}_{V}$. The dimensions of $\hat{N}_{V}$ and the matrices $K_{11}, K_{13}, K_{23}$ and $K_{33}$ are given in (22). Note that since the matrices $B_{i j}$ all lack the first row and column, the only non-zero part of $\hat{N}_{V}$ that we need to consider for definiteness is the condensed version (we neglect the rows and columns that consist of zeros) of the lower $3 \times 3$ block in $(22)$.

Let us denote the condensed version of the lower $3 \times 3$ block in $\hat{N}_{V}$ with $\tilde{N}$ and use a similar notation also for the rest of the matrices. That means that we should consider definiteness of

$$
\begin{aligned}
& \tilde{N}=\left[\begin{array}{ccc}
2 \alpha \tilde{K}_{11} & \mathbf{0}_{6,6} & \sqrt{2} \tilde{K}_{13} \\
\mathbf{0}_{6,6} & 2 \beta \tilde{K}_{11} & \sqrt{2} \tilde{K}_{23} \\
\sqrt{2} \tilde{K}_{13}^{T} & \sqrt{2} \tilde{K}_{23}^{T} & 2 \tilde{K}_{33}
\end{array}\right], \quad \tilde{K}_{33}=-(\Sigma+\Sigma)^{T} \\
& \tilde{K}_{11}=\left[\begin{array}{cc}
\tilde{B}_{11} & \tilde{B}_{12} \\
\tilde{B}_{21} & \tilde{B}_{22}
\end{array}\right], \quad \tilde{K}_{13}=\left[\begin{array}{c}
\left(\Delta-I_{3}\right) \tilde{B}_{11} \\
\left(\Delta-I_{3}\right) \tilde{B}_{12}
\end{array}\right], \quad \tilde{K}_{23}=\left[\begin{array}{c}
-\Delta \tilde{B}_{11} \\
-\Delta \tilde{B}_{12}
\end{array}\right] .
\end{aligned}
$$

Note again that we have now replaced all $4 \times 4$ matrices with the corresponding $3 \times 3$ ones. We have also kept the notation $\Delta$ and changed $\Sigma_{1 V}$ to $\Sigma$.

We find that a sufficient condition for positive semi-definiteness of $\tilde{N}$ is

$$
\tilde{K}_{11}>0 \text { and }-\left(\Sigma+\Sigma^{T}\right)=\tilde{K}_{33} \geq \frac{1}{2 \alpha} \tilde{K}_{13}^{T} \tilde{K}_{11}^{-1} \tilde{K}_{13}+\frac{1}{2 \beta} \tilde{K}_{23}^{T} \tilde{K}_{11}^{-1} \tilde{K}_{23},
$$

because we can factorize $\tilde{N}$ as $\tilde{N}=\varepsilon L D L^{T}$ with

$$
D=\left[\begin{array}{ccc}
2 \alpha \tilde{K}_{11} & \mathbf{0} & \mathbf{0} \\
\mathbf{0} & 2 \beta \tilde{K}_{11} & \mathbf{0} \\
\mathbf{0} & \mathbf{0} & D_{33}
\end{array}\right], \quad L=\left[\begin{array}{ccc}
I & \mathbf{0} & \mathbf{0} \\
\mathbf{0} & I & \mathbf{0} \\
\frac{1}{\sqrt{2} \alpha} \tilde{K}_{13}^{T} \tilde{K}_{11}^{-1} \frac{1}{\sqrt{2} \beta} \tilde{K}_{23}^{T} \tilde{K}_{11}^{-1} I
\end{array}\right] \text {, }
$$

and $D_{33}=2 \tilde{K}_{33}-\frac{1}{\alpha} \tilde{K}_{13}^{T} \tilde{K}_{11}^{-1} \tilde{K}_{13}-\frac{1}{\beta} \tilde{K}_{23}^{T} \tilde{K}_{11}^{-1} \tilde{K}_{23}$.

The conditions (19), (23) and (27) make the matrix $\hat{N}$ positive semi-definite, which implies that matrix $N$ is positive semi-definite, since for a arbitrary vector $\mathbf{y}$,

$$
\mathbf{y}^{T} N \mathbf{y}=\mathbf{y}^{T} S^{T} \hat{N} S \mathbf{y}=\hat{\mathbf{y}}^{T} \hat{N} \hat{\mathbf{y}} \geq 0 .
$$

The matrix $\tilde{K}_{11}^{-1}$ can be written in block matrix form as

$$
\tilde{K}_{11}^{-1}=\left[\begin{array}{cc}
\tilde{B}_{11}^{-1}+\tilde{B}_{11}^{-1} \tilde{B}_{12} \tilde{D}^{-1} \tilde{B}_{21} \tilde{B}_{11}^{-1} & -\tilde{B}_{11}^{-1} \tilde{B}_{12} \tilde{D}^{-1} \\
-\tilde{D}^{-1} \tilde{B}_{21} \tilde{B}_{11}^{-1} & \tilde{D}^{-1}
\end{array}\right],
$$

with $\tilde{D}=\tilde{B}_{22}-\tilde{B}_{21} \tilde{B}_{11}^{-1} \tilde{B}_{12}$. The choice $\Delta=\delta I_{3}(\delta \in \mathcal{R})$ simplifies the algebra considerably and leads to

$$
\tilde{K}_{13}^{T} \tilde{K}_{11}^{-1} \tilde{K}_{13}^{T}=(1-\delta)^{2} \tilde{B}_{11}, \quad \text { and } \quad \tilde{K}_{23}^{T} \tilde{K}_{11}^{-1} \tilde{K}_{23}^{T}=\delta^{2} \tilde{B}_{11}
$$


That means that the last condition in (27) together with the assumption that $\Sigma$ is symmetric leads to

$$
\Sigma \leq-\frac{\left[\beta(1-\delta)^{2}+\alpha \delta^{2}\right] \varepsilon}{4 \alpha \beta} \tilde{B}_{11}
$$

It is easy to verify that the right hand side of (29) has the least restrictive value $-\varepsilon \tilde{B}_{11} /(4(\alpha+\beta))$ when $\delta=\beta /(\alpha+\beta)$.

Now we have done all the necessary derivations and we can summarize the result in the following proposition.

Proposition 5.1 If the conditions

$$
\begin{aligned}
\Sigma_{1 I}^{L} & \leq A_{1}^{-} / 2, & & \text { (inviscid stability) } \\
\Sigma_{1 V}^{L} & \leq-\varepsilon \tilde{B}_{11} / 4(\alpha+\beta), & & \text { (viscous stability) } \\
\Sigma_{2}^{L} & =-\varepsilon \beta I_{4} /(\alpha+\beta), & & \text { (viscous stability) } \\
\Sigma_{1}^{R} & =\Sigma_{1}^{L}-A_{1}, & & \text { (inviscid conservation) } \\
\Sigma_{2}^{R} & =\Sigma_{2}^{L}+\varepsilon I_{4} . & & \text { (viscous conservation) }
\end{aligned}
$$

are satisfied, then the scheme (15) is stable and conservative.

Remark Recall that $\alpha=\alpha^{L} p^{L}$ and $\beta=\beta^{R} p^{R}\left(0 \leq \alpha^{L}, \beta^{R} \leq 1\right)$ where

$$
p^{L}=\Delta x^{L} \cdot \begin{cases}\frac{1}{2} & \text { 2nd order SBP, } \\
\frac{77}{48} & 4 \text { th order SBP, } \quad p^{R}=\Delta x^{R} \cdot\left\{\begin{array}{ll}
\frac{1}{2} & \text { 2nd order SBP } \\
\frac{17}{43649} & \text { 6th order SBP }
\end{array} \quad\right. \text { 4th order SBP } \\
\frac{13649}{43200} & 6 \text { th order SBP }\end{cases}
$$

In order to limit the spectral radius of the problem, the values of $\alpha^{L}$ and $\beta^{R}$ should be chosen as large as possible, that is $\alpha^{L}=\beta^{R}=1$.

Remark Note again that the conservation conditions are a subset of the total number of conditions required for stability.

\section{$6 \quad$ Numerical experiments}

The derivation of the stability and conservation properties as expressed in Proposition 5.1 was done for the constant coefficient problem. We now verify that the result of the linear analysis is valid for the full non-linear Navier-Stokes equations.

\subsection{Verification of accuracy and stability for the Navier-Stokes equations}

We consider a calculation on two sub-domains coupled at an interface, see Figure 1. A stationary viscous shock problem where the middle of shock is located at the 
interface is calculated. This problem has an analytical solution (for Prandtl number $\operatorname{Pr}=3 / 4$ ) which means that we have full control of the errors. The Mach number in front of the shock in the reference frame of the shock is 2.0 and the angle of the shock relative to the Cartesian frame is $15^{\circ}$. The Reynolds number $R e=50.0$ is based on the Mach number of shock. The penalty terms in (30) are chosen by the minimum required values. We integrate the solution to steady-state using the third order low storage explicit time advancement scheme of Le and Moin [9].

In the hybrid scheme, the second derivative SBP operator is constructed with $2 p$-th $(p=1,2, \ldots)$ order accuracy internal and $(p-1)$-th order at the boundary by using a diagonal norm. It was proved in [20] that if the solution is pointwise bounded, the accuracy of the scheme is two orders higher than the accuracy of the second derivative approximation at the boundaries. The convergence rates for the second-, fourth-, sixth- and eighth- order schemes are thus 2, 3, 4 and 5, respectively. Since the errors for all variables (density, velocities and energy) are very similar, only the density errors are shown in our calculations. The accuracy is shown in Tables 1 and Table 2 . The results are in agreement with the theory, see $[4,5,20]$.

\begin{tabular}{|c|c|c|c|c|c|c|c|c|}
\hline \multirow[b]{2}{*}{ Points/Block } & \multicolumn{2}{|c|}{ 2nd order } & \multicolumn{2}{|c|}{4 th order } & \multicolumn{2}{|c|}{6 th order } & \multicolumn{2}{|c|}{ 8th order } \\
\hline & Err & $q$ & Err & $q$ & Err & $q$ & Err & $q$ \\
\hline $17 \times 17$ & -1.29 & - & -1.47 & - & -1.14 & - & - & - \\
\hline $33 \times 33$ & -1.89 & 1.99 & -2.24 & 2.56 & -1.90 & 2.52 & -1.89 & - \\
\hline $65 \times 65$ & -2.55 & 2.18 & -3.14 & 3.00 & -2.92 & 3.41 & -3.03 & 3.77 \\
\hline $129 \times 129$ & -3.18 & 2.11 & -4.12 & 3.24 & -4.01 & 3.59 & -4.39 & 4.53 \\
\hline $257 \times 257$ & -3.80 & 2.03 & -5.06 & 3.15 & -5.11 & 3.66 & -5.90 & 5.01 \\
\hline
\end{tabular}

The convergence rates of density on two uniform sub-domains.

\begin{tabular}{|c|c|c|c|c|c|c|c|c|}
\hline \multirow{2}{*}{$\begin{array}{c}\text { Points } \\
\text { (left)+(right) }\end{array}$} & \multicolumn{2}{|c|}{ 2nd order } & \multicolumn{2}{|c|}{4 th order } & \multicolumn{2}{|c|}{6 th order } & \multicolumn{2}{|c|}{ 8th order } \\
\hline & Err & $q$ & Err & q & Err & $q$ & Err & q \\
\hline $33 \times 33+17 \times 33$ & -1.43 & - & - & - & -1.30 & - & -1.39 & - \\
\hline $65 \times 65+33 \times 65$ & -2.02 & 1.94 & -2.35 & 2.59 & -2.06 & 2.52 & -2.04 & - \\
\hline $129 \times 129+65 \times 129$ & -2.65 & 2.09 & -3.23 & 2.91 & -3.02 & 3.21 & -3.14 & 3.68 \\
\hline $257 \times 257+129 \times 257$ & -3.26 & 2.05 & -4.13 & 2.98 & -4.09 & 3.53 & -4.48 & 4.43 \\
\hline $513 \times 513+257 \times 513$ & -3.88 & 2.05 & -5.02 & 2.98 & -5.22 & 3.76 & -5.92 & 4.80 \\
\hline
\end{tabular}

Table 2

The convergence rates of density on two non-uniform subdomains.

In the next calculation we consider the solution computed on the mesh in Figure 1. Figure 2(a) shows the density isolines using the 4 th order discretization. The 
corresponding cut at $y=0$ can be found in Figure 2(b). The distribution of density close to the interface $x=0$ is very smooth, which illustrates that the interface does not introduce large reflections and oscillations.

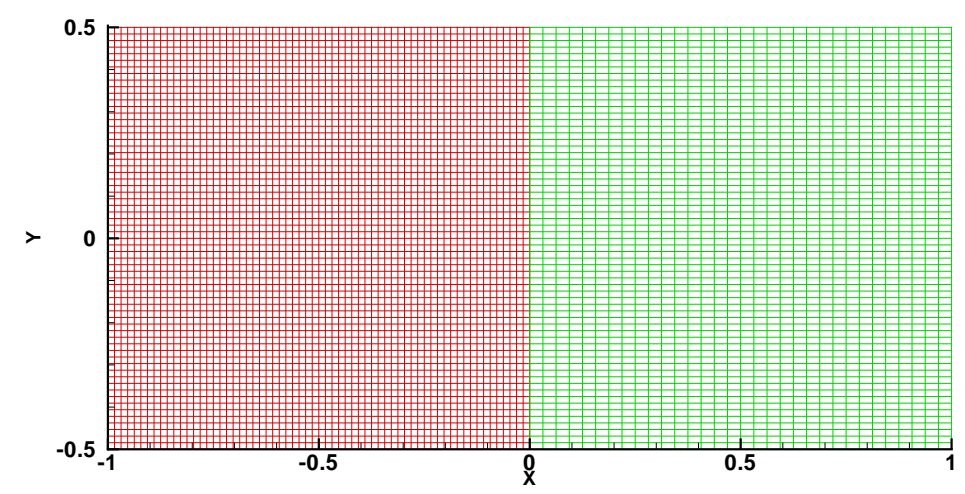

Fig. 1. A hybrid mesh of $65 \times 65+33 \times 65$ grid points.

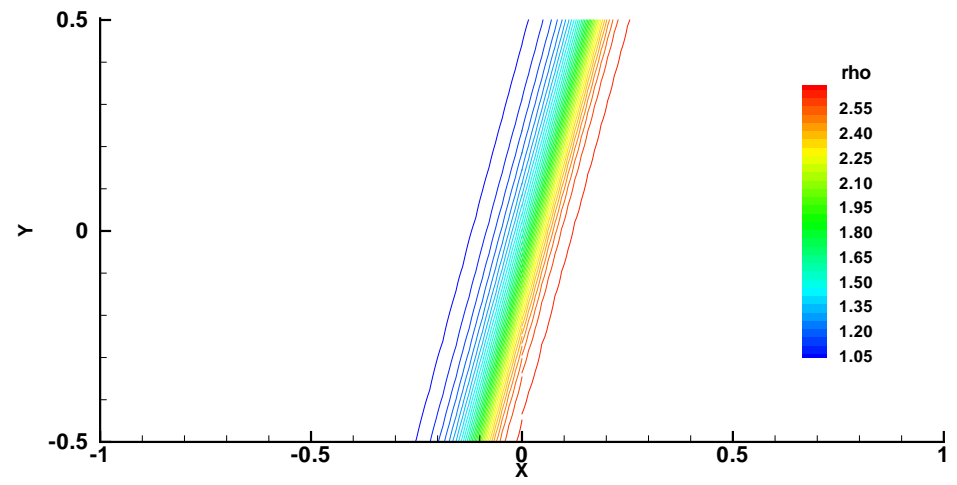

(a) The whole computational domain

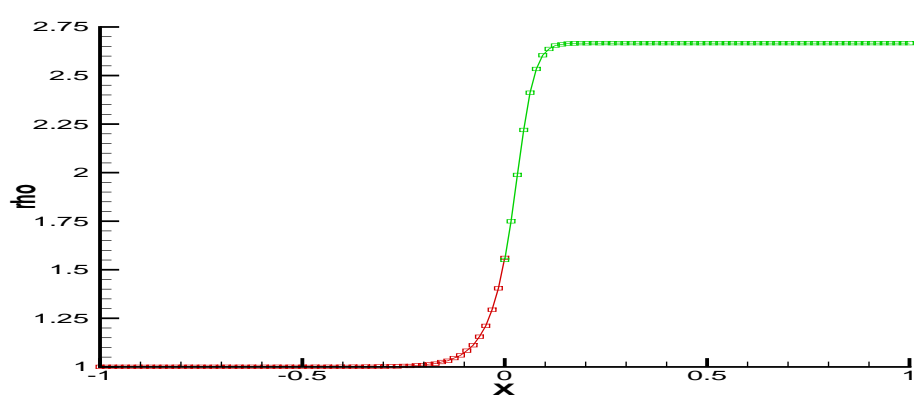

(b) A cut at $y=0$

Fig. 2. Density isolines for a 4 th order calculation. $65 \times 65$ grid points are used in both subdomains.

The density errors at $y=0$ with SBP operators of different order are shown in Figures 3 and 4 . Figure 3 shows the result for two uniform meshes, while in Figure 


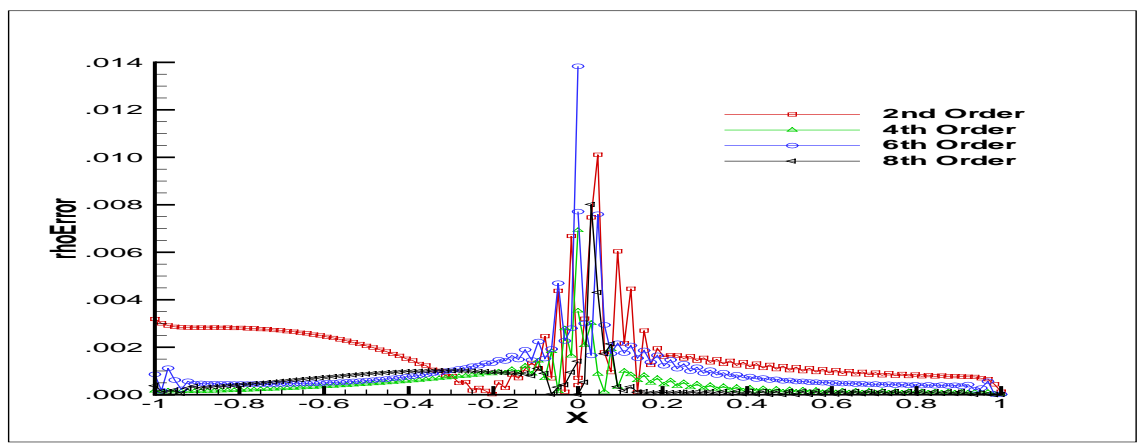

(a) $65 \times 65$ points/block

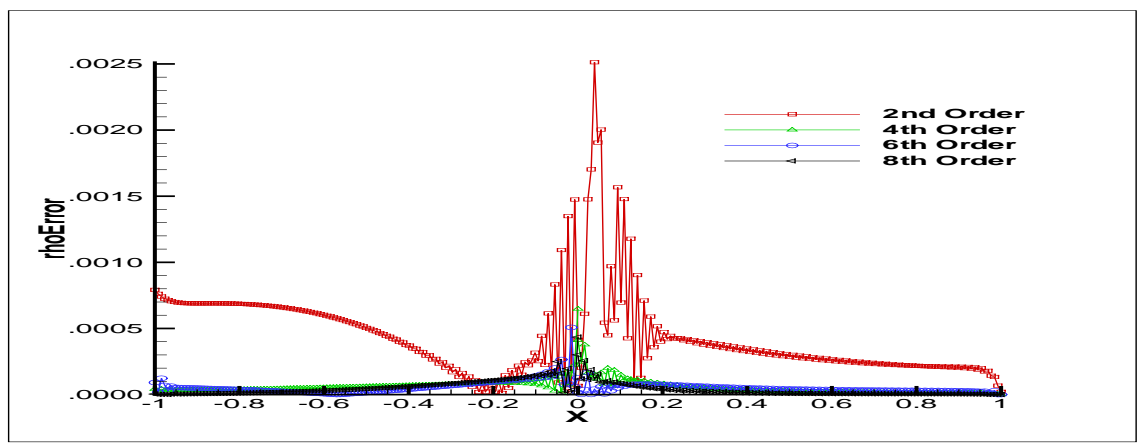

(b) $129 \times 129$ points/block

Fig. 3. The errors in density at $y=0$ with SBP operators of different orders.

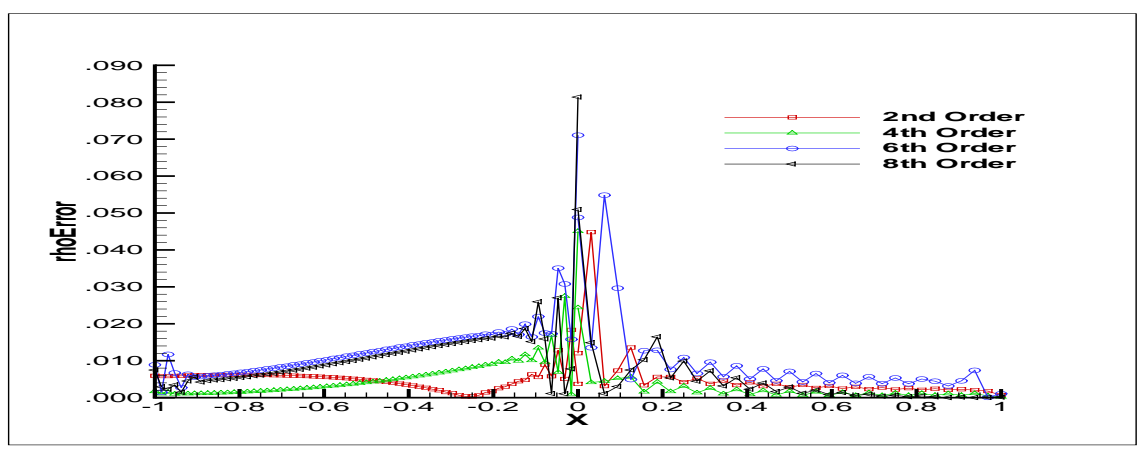

(a) $65 \times 65+33 \times 65$

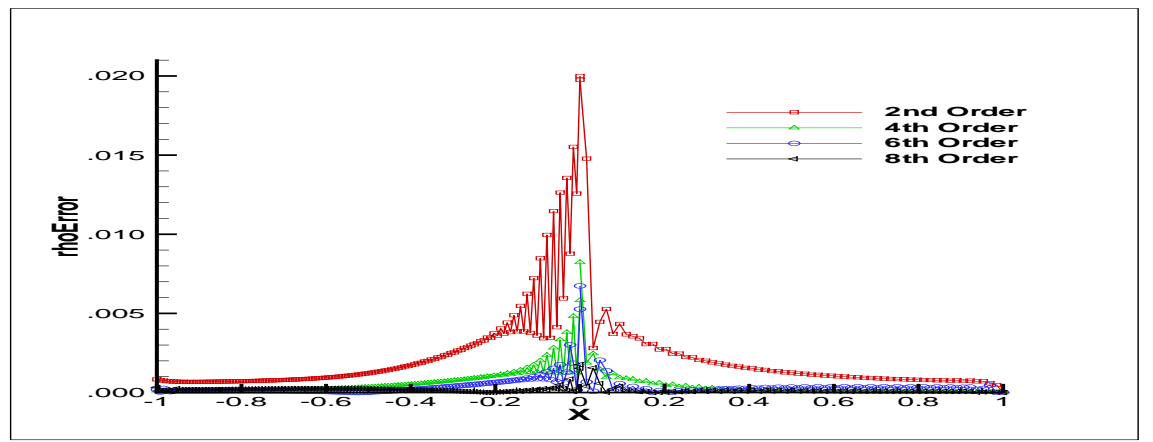

(b) $129 \times 129+65 \times 129$ points/block

Fig. 4. The errors in density at $y=0$ with SBP operators of different orders. 
4 the right block is twice as coarse in the $x$-direction as the left block. Figures 3(a) and 4(a) show that the higher order schemes have rather large errors, comparable to the lower order schemes close to the interface $x=0$ for the coarse mesh. However, when the mesh is refined, $(129 \times 129$ and $65 \times 129$, respectively $)$ the higher order schemes outperform the lower order schemes (see Figures 3(b) and 4(b)). Tables 1-2 and Figures 3-4 illustrate that the interface treatment is stable and accurate for all orders of accuracy.

To further illustrate the necessity of having correct penalty terms we neglect the viscous penalty term completely. This leads to a complete failure for all schemes (blow up in a couple of time steps), see Figure 5.

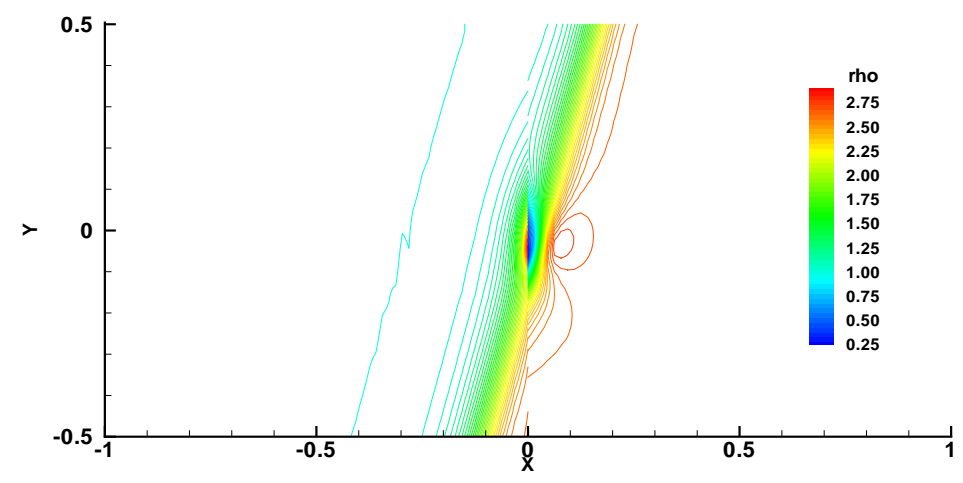

(a) The density

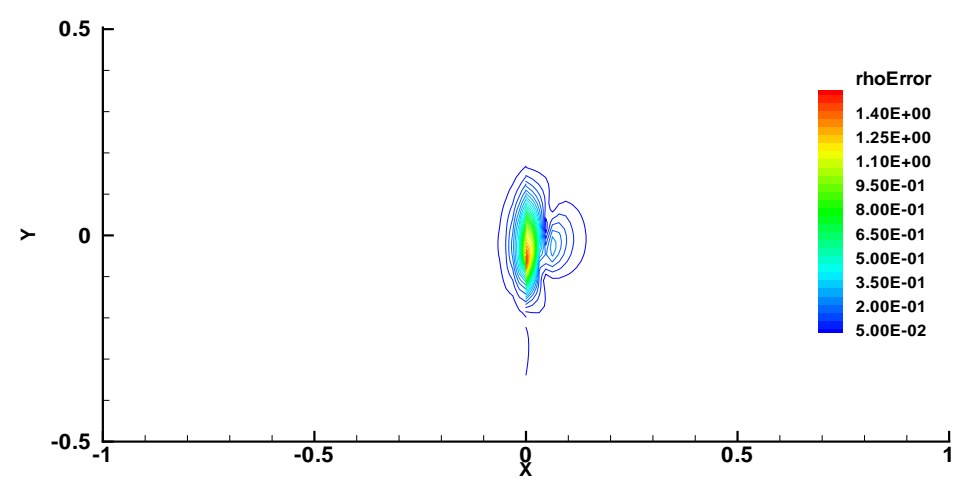

(b) The error in density

Fig. 5. A 4th order calculation without the necessary viscous penalty terms.

Finally, we demonstrate the multi-block method on a moving shock problem. The unsteady computation has been carried out on a uniform grid of $65 \times 65$ in each block in combination with the the 4 th oder accurate SBP operator. All penalty parameters have the same values as for the previous steady case. The shock moves at Mach $=0.15$ under $45^{\circ}$ degrees. Snapshots of the solution between $t=0.0$ and $t=8.0$ are shown in Figure 6 . The shape of the shock through the interface $x=0$ remains intact, and the corresponding errors are small, see Figure 7. 

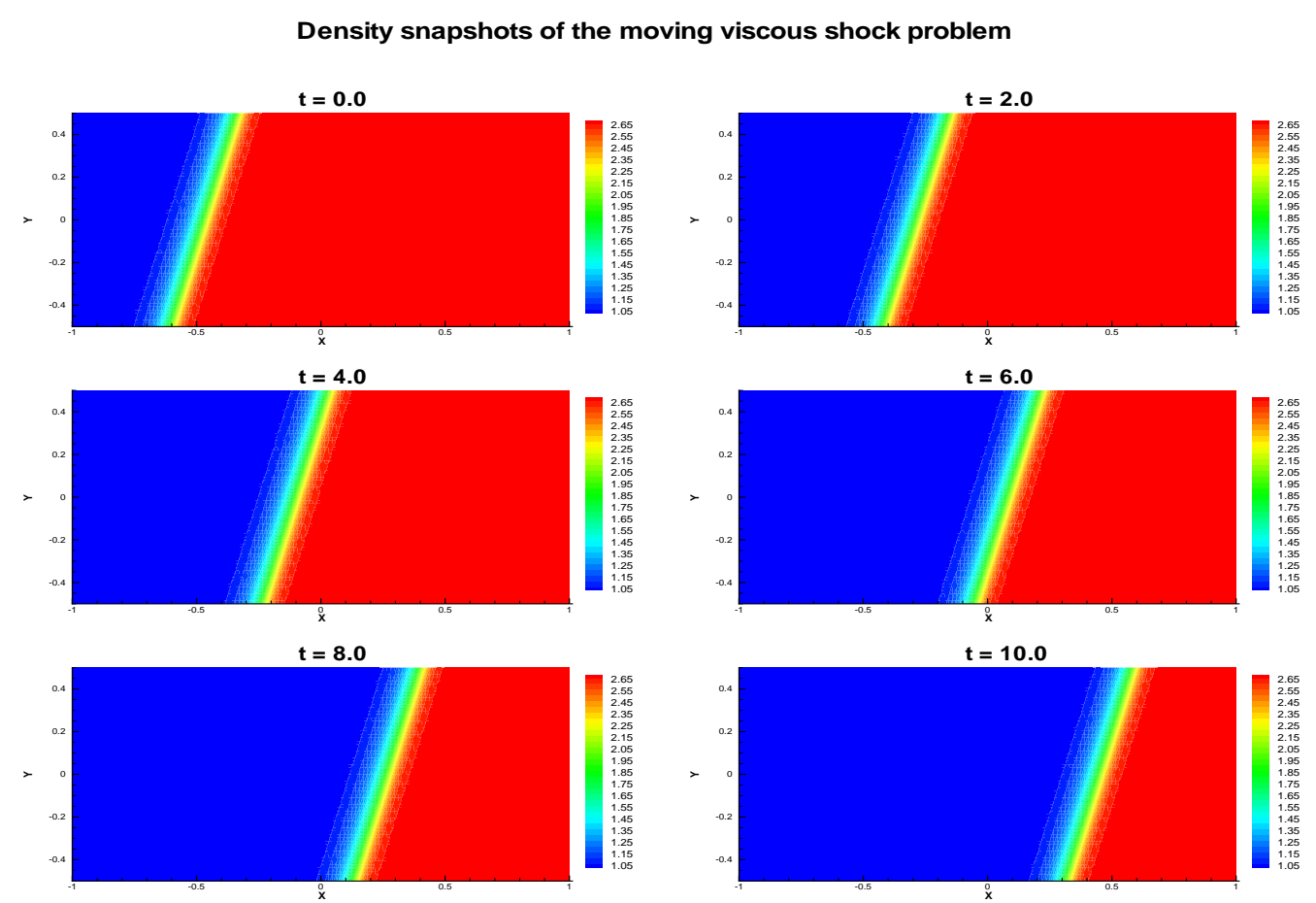

Fig. 6. Density isolines, 4th order accuracy for the unsteady shock problem.

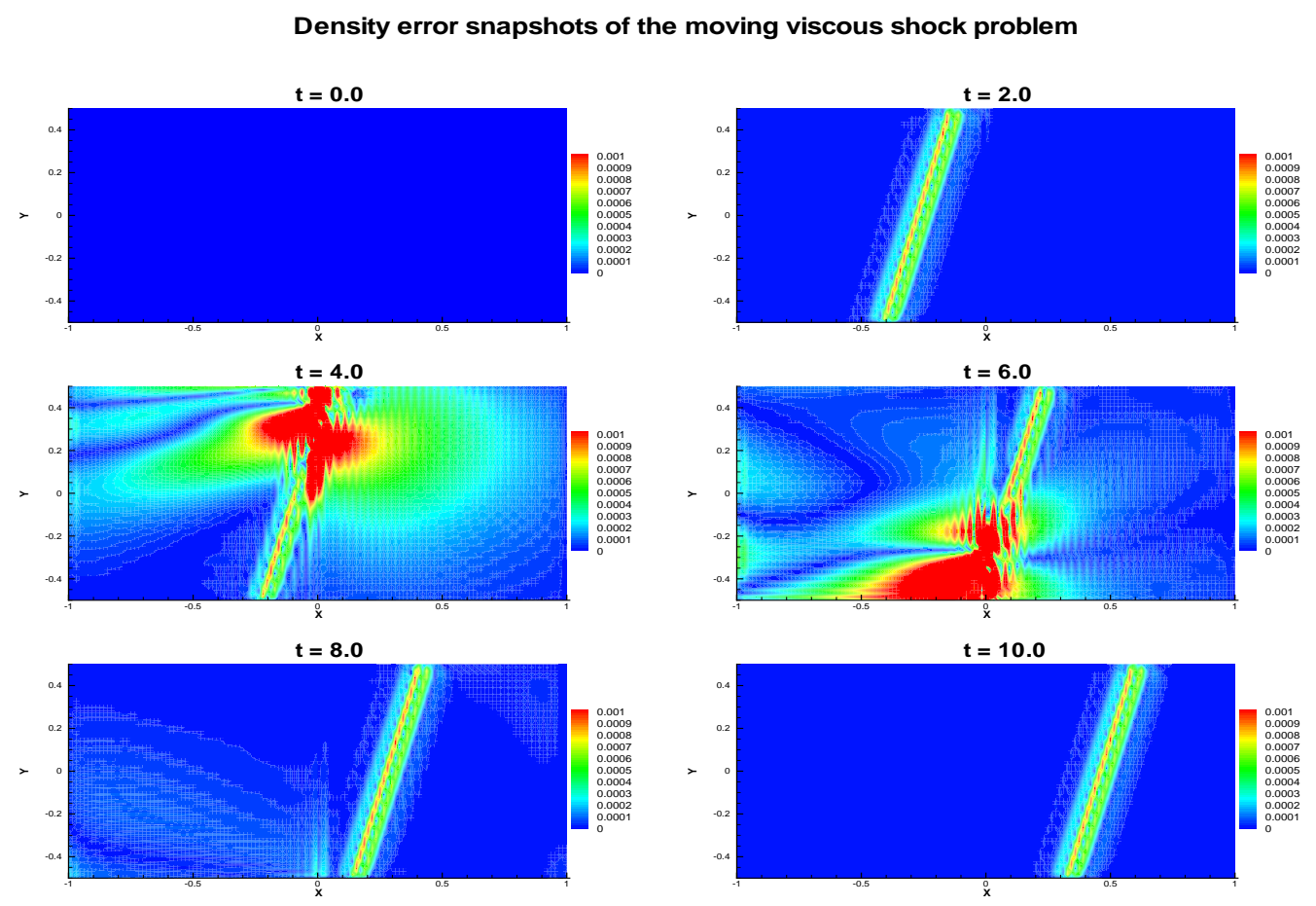

Fig. 7. The error in density, 4th order accuracy for the unsteady shock problem. 


\section{Conclusions}

We have proved stability and conservation of a high order accurate multi-block finite difference method applied to the Navier-Stokes equations. As theoretical tools we have used difference operators of SBP type, a penalty technique for the interface conditions and the energy method.

The stability and conservation conditions are derived without approximations. This indicates that the derived conditions are sharp. That conclusion is supported by the numerical calculations which show that instabilities occur if the conditions are violated.

Mesh refinement studies for a steady viscous shock and computations of a moving viscous shock has been performed. The numerical experiments support the theoretical conclusions and show that the interface coupling is stable and converge at the correct order.

\section{References}

[1] S. Abarbanel and D. Gottlieb. Optimal time splitting for two- and threedimensional Navier-Stokes equations with mixed derivatives. Journal of Computational Physics, 41:1-43, 1981.

[2] M.H. Carpenter, J. Nordström, and D. Gottlieb. A stable and conservative interface treatment of arbitrary spatial accuracy. Journal of Computational Physics, 148:341-365, 1999.

[3] J. Gong and J. Nordström. A stable and efficient hybrid method for viscous problems in complex geometries. Journal of Computational Physics, 226(2):1291-1309, 2007.

[4] B. Gustafsson. The convergence rate for difference approximation to mixed initial boundary value problems. Mathematics of Computation, 29, 1975.

[5] B. Gustafsson. The convergence rate for difference approximation to general mixed initial boundary value problems. SIAM Journal on Numerical Analysis, 18(2):179-190, 1981.

[6] B. Gustafsson, H.-O. Kreiss, and J. Oliger. Time Dependent Problems and Difference Methods. John Wiley \& Sons, Inc., 1995.

[7] R. A. Horn and C. R. Johnson. Topics in Matrix Analysis. Cambridge University Press, 1991.

[8] H.-O. Kreiss and G. Scherer. Finite element and finite difference methods for hyperbolic partial differential equations, in: C. De Boor (Ed.), Mathematical Aspects of Finite Elements in Partial Differential Equation. Academic Press, New York, 1974. 
[9] H. Le and P. Moin. An improvement of fractional step methods for the incompressible Navier-Stokes equations. Journal of Computational Physics, 92:369-379, 1991.

[10] K. Mattsson and J. Nordström. Summation by parts operators for finite difference approximations of second derivatives. Journal of Computational Physics, 199:503-540, 2004.

[11] J. Nordström and M. H. Carpenter. Boundary and interface conditions for high order finite difference methods applied to the Euler and Navier-Stokes equations. Journal of Computational Physics, 148:621-645, 1999.

[12] J. Nordström and M. H. Carpenter. High-order finite difference methods, multidimensional linear problems and curvilinear coordinates. Journal of Computational Physics, 173:149-174, 2001.

[13] J. Nordström and J. Gong. A stable hybrid method for hyperbolic problems. Journal of Computational Physics, 212:436-453, 2006.

[14] J. Nordström and R. Gustafsson. High order finite difference approximations of electromagnetic wave propagation close to material discontinuities. Journal of Scientific Computing, 18(2):215-234, 2003.

[15] J. Nordström, F. Ham, M. Shoeybi, E. van der Weide, M. Svärd, K. Mattsson, G. Iaccarino, and J. Gong. A hybrid method for unsteady fluid flow. In press in Computers and Fluids, 2009.

[16] J. Nordström and M. Svärd. Well-posed boundary conditions for the NavierStokes equations. SIAM Journal on Numerical Analysis, 43(3):1231-1255, 2005.

[17] B. Strand. Summation by parts for finite difference approximation for $\mathrm{d} / \mathrm{dx}$. Journal of Computational Physics, 110(1):47-67, 1994.

[18] J.C. Strickwerda. Initial boundary value problems for incompletely parabolic systems. Commun. Pure Appl. Math., 9(3):797-822, 1977.

[19] M. Svärd, M.H. Carpenter, and J. Nordström. A stable high-order finite difference scheme for the compressible Navier-Stokes equations: far-field boundary conditions. Journal of Computational Physics, 225(1):1020-1038, 2007.

[20] M. Svärd and J. Nordström. On the order of accuracy for difference approximations of initial-boundary value problems. Journal of Computational Physics, 218(1):333-352, 2006.

[21] M. Svärd and J. Nordström. A stable high-order finite difference scheme for the compressible Navier-Stokes equations: No-slip wall boundary conditions. Journal of Computational Physics, 227(10):4805-4824, 2008. 